from competition with other species, from simple physical constraints or from both?

Populations do sometimes colonize new areas, with a habitat more or less different from the old one; what are the genetic and ecological consequences? Do new species often form as a result of the drastic changes experienced during a colonization event? Is the continual process of extinction and recolonization more important than simple diffusion in spreading genes through a population (singly or in combination), as envisaged by Wright in his "shifting balance" theory? Are the proportions of different species at an equilibrium resulting from steady competitive pressure, or can community structure only be understood in terms of continual, stochastic turnover?

Given the range of these questions, all of which might be considered under the title The Evolutionary Biology of Colonizing Species, this book is disappointingly narrow in scope. Although Parsons sets out to "integrate population genetics and ecology in order to develop a more coherent theory of evolutionary biology", a laudable aim, his book is almost entirely confined to considering the response of a few species, each considered in isolation to the physical environment. It was Darwin's view that "the range of the inhabitants of any country by no means exclusively depends on insensibly changing conditions, but in large part on the presence of other species"'; without considering interactions between species, one can hardly understand their ecology.

Nearly all species considered by Parsons are in the genus Drosophila. A great deal of information is presented comparing the ranges of different Drosophila species in the wild to their responses to physical stresses in the laboratory (alcohol vapour or extremes of temperature, for example). Although this comparative method can be fruitful, we may need a better understanding of the relationship between laboratory and field conditions, in a larger number of species, before the results can be very informative.

A more fundamental criticism of the book is that difficult distinctions, such as those between "hard" and "soft" selection, "structural" and "regulatory" genes, "genic" or "organismic" selection, and "peripheral" and "central" populations, are introduced without any clear explanation of their meaning. This is not only perplexing for a reader unfamiliar with the terminology, but also makes the discussion disturbingly vague; the difficulty of testing many of the central questions against field data makes it especially important to frame such questions unambiguously. Understanding the evolutionary biology of colonizing species will certainly require a synthesis of population genetics and ecology; but that synthesis remains to be achieved.

Nick Barton is in the Department of Genetics and Biometry at University College London.

\section{Atmospheres around the Solar System}

\section{Conway B. Leovy}

The Origin and Evolution of Planetary

Atmospheres.

By Ann Henderson-Sellers.

Adam Hilger, Bristol/Heyden, Phila-

delphia: 1983. Pp.236. £19.50, \$34.

WHY is Earth unique among the planetary bodies of the Solar System in providing an environment suitable for life? Why has its climate remained stable over the past three or four billion years despite probable variations in solar output and possible cometary or asteroidal collisons? How likely is it that stars other than the Sun possess habitable zones? Such far-reaching questions have been stimulated by the comparative study of planetary atmospheres and are addressed by Ann Henderson-Sellers in this survey of a complex and highly interdisciplinary field.

All of the planetary bodies in the Solar System are considered, including the major satellites which do not now have atmospheres. Only the outer planets, from Jupiter to Neptune, have primary atmospheres little influenced by such evolutionary processes as surface interactions and escape. Titan is a fascinating special case, with a slowly evolving atmosphere of nitrogen, argon and hydrocarbons.

The questions most relevant to the existence of life centre around the role and

\section{Hunt for nutrition}

\section{Diane Gifford-Gonzalez}

Bison Kills and Bone Counts: Decision

Making by Ancient Hunters.

By John D. Speth.

University of Chicago Press: 1983.

Pp.227. Hbk \$20, f17;

pbk \$8, £6.80.

OCCASIONALLY, a single study, by drawing together hitherto isolated areas of research, invites scientists in a discipline to take their work to a new level of synthesis and discourse. John Speth's Bison Kills and Bone Counts extends such an invitation to zooarchaeologists and to everyone who uses faunal evidence from archaeological sites to reconstruct past human diet. The book's subtitle, Decision Making by Ancient Hunters, signals its principal aim: to reconstruct the human decisions and actions that created an archaeological site, its internal structure and the patterning in its faunal remains. To this end, Speth applies the past decade's findings in vertebrate taphonomy and lithic replication studies to reconstruct the train of events that formed the Garnsey fate of water on the terrestrial planets. Of these, Venus appears to have undergone catastrophic heating at an early stage, leading to evaporative loss of what may have been a rich initial endowment of water. Water has apparently been an important agent of modification of the Martian surface, though geological interpretation of spacecraft images by Michael Carr and others, and surface adsorption models of Fraser Fanale suggest that liquid water may not have been widespread for at least the past three billion years. Nevertheless, large fluctuations in surface pressure and climate may occur even now. Earth's climatic stability is all the more impressive in the context of its neighbours' catastrophic and fluctuating climates, and Henderson-Sellers focuses on the processes which control planetary surface temperatures, arguing that Earth's unique hydrosphere may be primarily responsible for climatic stability.

Those seeking a self-contained overview of the field will be disappointed. In many cases, the explanations of the physical and chemical controls on atmospheric evolution and climate are obscure or incomplete, and one is frequently taken around issues rather than to their core. Nevertheless, the sweeping perspective and interdisciplinary scope of this volume will reward the careful reader by calling to mind a host of intriguing problems worthy of further exploration.

Conway B. Leovy is in the Department of Atmospheric Sciences at the University of Washington, Seattle.

site, a fifteenth-century bison-kill complex in New Mexico. He then draws upon ethnography, ethnoarchaeology and nutritional research to build a case for nutritionally conditioned selectivity on the part of the bison hunters in their butchery and use of male rather than female bison.

The distribution of bones and lithics along a small tributary of the Pecos River reflects successive kill and butchery episodes, spanning nearly a century. These kills, according to Michael Wilson's analysis of the bison's dentitions, took place in the spring. From sex ratios of crania, Speth suggests that the hunters focused on "bull groups", the usual social grouping of male bison except during rut. He explains the presence of females in bone clusters supposedly representing bullgroup kills by noting that non-calving females join such associations among modern bison, although a kill of at least one cow-calf group, also in the spring, is implied by the presence of some calf remains.

Speth applies recent findings from vertebrate taphonomy in analysing the patterns of bone representation, state of preservation and damage patterns of the bison remains, inferring that the assemblage has been minimally affected by carnivores or 